\title{
Integrated Nutrient Management (INM) on Nutrient Availability, Uptake and Yield of Tomato (Lycopersicon esculentum Mill.) cv. "Gujrat Tomato-2"
}

\author{
G. S. Tekale ${ }^{1}$, S. N. Saravaiya ${ }^{1}$, P. B. Jadhav ${ }^{2}$, C. D. Tekale ${ }^{3}$ and R. P. Patel ${ }^{4}$ \\ ${ }^{1}$ Regional Horticultural Research Station, Department of Vegetable Science, \\ ASPEE College of Horticulture and Forestry, Navsari Agricultural University, \\ Navsari-396 450, Gujarat, India \\ ${ }^{2}$ Agricultural Research Expert, Ecofrost Technologies Pvt. Ltd., Survey No 134/1, 134/2,130/3, \\ Jeevan Nagar, Tathawade, Pune, Maharashtra- 411 033, India \\ ${ }^{3}$ Department of Agronomy,Mahatma Phule Krushi Vidyapeeth, Rahuri, Maharashtra, India \\ ${ }^{4}$ Horticulture Officer, Government of Gujarat, India \\ *Corresponding author: ganeshstekale@gmail.com
}

\section{A B S T R A C T}

\begin{tabular}{|l|}
\hline Ke y w o r d s \\
INM, Nutrient \\
Availability, \\
Uptake and \\
Yield of Tomato, \\
Zinc Sulphate, \\
Ferrous Sulphate. \\
\hline Article Info \\
\hline $\begin{array}{l}\text { Accepted: } \\
\text { 04 April } 2017 \\
\text { Available Online: } \\
\text { 10 May } 2017\end{array}$ \\
\hline
\end{tabular}

A field experiment was carried out to study the "Integrated nutrient management (INM) on availability of nutrients in soil, nutrient uptake and yield of tomato (Lycopersicon esculentum Mill.) cv. Gujarat Tomato-2"during rabi season of 2011-12 and 2012-13 at the Regional Horticultural Research Station, Navsari Agricultural University, Navsari, Gujarat, India. The experiment was conducted on fixed plot site with a set of twelve treatments. Among different INM treatments, FYM $20 \mathrm{t} \mathrm{ha}^{-1}+100 \% \mathrm{RDF}$ had significantly the highest available $\mathrm{N}\left(255,259\right.$ and $\left.257 \mathrm{~kg} \mathrm{ha}^{-1}\right), \mathrm{P}_{2} \mathrm{O}_{5}(63.5,61.9$ and 62.7 $\left.\mathrm{kg} \mathrm{ha}^{-1}\right), \mathrm{K}_{2} \mathrm{O}\left(327,322\right.$ and $\left.325 \mathrm{~kg} \mathrm{ha}^{-1}\right)$ nutrient in soil after harvest, $\mathrm{N}(1.54,1.56$ and $1.55 \%), \mathrm{P}_{2} \mathrm{O}_{5}(0.49,0.50$ and $0.50 \%), \mathrm{K}_{2} \mathrm{O}(1.30,1.33$ and $1.32 \%)$ nutrient content in tomato plant, total nutrient uptake $\mathrm{N}\left(136,145\right.$ and $\left.140 \mathrm{~kg} \mathrm{ha}^{-1}\right), \mathrm{P}(45.2,49.8$ and $47.5 \mathrm{~kg}$ $\left.\mathrm{ha}^{-1}\right), \mathrm{K}\left(66,76\right.$ and $\left.71 \mathrm{~kg} \mathrm{ha}^{-1}\right)$, fruit yield plant ${ }^{-1}(1.49,1.58$ and $1.54 \mathrm{~kg})$ and fruit yield $\operatorname{plot}^{-1}(29.86,31.56$ and $30.71 \mathrm{~kg})$ during 2011-12, 2012-13 and pooled analysis. So far nutrients uptake was concerned, remarkable increase in values of total uptake of nutrients viz., N, P and $\mathrm{K}$ by tomato in the treatment combination of $100 \% \mathrm{RDF}+\mathrm{FYM} 20 \mathrm{tha}^{-1}$ $\left(\mathrm{T}_{1}\right)$. Different INM treatments had non-significant effects on $\mathrm{N}, \mathrm{P}$ and $\mathrm{K}$ contents.

\section{Introduction}

Tomato is one of the most common, leading, widely consumed, popular, staple, day neutral, self pollinated, annual and economically important solanaceous fruit vegetable crop. Its fruits are very popular among people of all social strata and consumed in variety of ways. It is equally liked by both poor and rich and is quite high in nutritive value. Apart from this, it also embodies certain Ayurvedic medicinal properties.
With the increasing population, the cultivable land resource is shrinking day to day. To meet the food, fiber, fuel, fodder and other needs of the growing population, the productivity of agricultural land and soil health needs to be improved.

The ocular evidence is that the nutrient management produces more food than nutrient levels. Therefore, it is obligatory to manage the nutrients in such a way that one 
can harvest good yield without deteriorating soil health. Scientific management of these sources is very an important for maintaining the soil productivity, to enhance fertilizer use efficiency and secure optimum vegetable production without harming the environment. Besides sustainable agricultural production, all round improvement in physical, chemical and biological make up of soils is the main aim of INM (Chadha, 2002).

Information on the conjoint use of organic manures, chemical fertilizers and micronutrients in tomato under the Indian conditions in general and the South Gujarat conditions in particular is very limited. Looking to the importance, future scope and a heavy demand of tomato by all class of consumers and also keeping in view of reducing the dose of $\mathrm{N}, \mathrm{P}$ and $\mathrm{K}$, a field trial was conducted.

\section{Materials and Methods}

The experiment entitled "Integrated nutrient management (INM) for tomato (Lycopersicon esculentum Mill.) $c v$. Gujarat Tomato-2" was carried out during the winter ( $r a b i)$ season of 2011-12 and 2012-13 at the Regional Horticultural Research Station (RHRS) of the Navsari Agricultural University, Navsari, Gujarat, India. The experiment was conducted on fixed plot site with a set of twelve treatments viz., $\mathrm{T}_{1}$ (FYM $20 \mathrm{t} \mathrm{ha}^{-1}+100 \%$ RDF) (75: 37.5: 62.5 NPK kg ha $\left.{ }^{-1}\right), \mathrm{T}_{2}$ (Farm Yard Manure (FYM) $20 \mathrm{t} \mathrm{ha}^{-1}$ ), $\mathrm{T}_{3}$ (Neem cake (NC) $\left.5 \mathrm{t} \mathrm{ha}^{-1}\right), \mathrm{T}_{4}$ (Vermicompost (VC) 5 $\left.\mathrm{t} \mathrm{ha}^{-1}\right), \mathrm{T}_{5}(75 \% \mathrm{RDF}+$ Farm Yard Manure $\left.20 \mathrm{t} \mathrm{ha}^{-1}\right), \mathrm{T}_{6}(75 \% \mathrm{RDF}+$ Neem cake $5 \mathrm{t}$ ha $\left.{ }^{1}\right), \mathrm{T}_{7}\left(75 \% \mathrm{RDF}+\right.$ Vermicompost $\left.5 \mathrm{tha}^{-1}\right), \mathrm{T}_{8}$ $\left(50 \% \mathrm{RDF}+\right.$ Farm Yard Manure $\left.20 \mathrm{t} \mathrm{ha}^{-1}\right), \mathrm{T}_{9}$ $\left(50 \% \mathrm{RDF}+\right.$ Neem cake $\left.5 \mathrm{t} \mathrm{ha}^{-1}\right), \mathrm{T}_{10}(50 \%$ $\mathrm{RDF}+$ Vermicompost $\left.5 \mathrm{t} \mathrm{ha}^{-1}\right), \mathrm{T}_{11}(100 \%$ $\left.\mathrm{RDF}+\mathrm{ZnSO}_{4} @ 25 \mathrm{~kg} \mathrm{ha}^{-1}\right)$ and $\mathrm{T}_{12}(100 \%$ $\left.\mathrm{RDF}+\mathrm{FeSO}_{4} @ 50 \mathrm{~kg} \mathrm{ha}^{-1}\right)$. The experiment was evaluated in Randomized Block Design
(RBD) (Panse and Sukhatme, 1986). Entire quantity of well rotten bulky organic manures (FYM, NC and VC) was manually applied and thoroughly incorporated into the respective plots as per the treatments before one month of transplanting. Twenty four days old, stocky and healthy seedlings of $15 \mathrm{~cm}$ height with 3 to 4 leaves, free from any insect pest and disease and true to type seedlings of 'GT-2' cultivar of tomato were selected and transplanted in the experimental field after dipping their roots in Imidachloprid solution for 15 minutes during both the years of winter seasons at $60 \mathrm{~cm} \times 60 \mathrm{~cm}$ apart in the late afternoon.

\section{Results and Discussion}

\section{Available Nutrient}

Data related to available $\mathrm{N}, \mathrm{P}_{2} \mathrm{O}_{5}$ and $\mathrm{K}_{2}$ Oin soil after harvesting as influenced by different INM treatments are given in Table2.Focusing on the research results of first year trial (2011-12), T 1 (FYM $\left.20 \mathrm{t} \mathrm{ha}^{-1}+100 \% \mathrm{RDF}\right)$ recorded the highest available ' $\mathrm{N}$ ' i.e.; $255 \mathrm{~kg}$ $\mathrm{ha}^{-1}$ which was at par with $\mathrm{T}_{5}$ and $\mathrm{T}_{8}$. The lowest status of available ' $\mathrm{N}$ ' in soil $(161 \mathrm{~kg}$ $\mathrm{ha}^{-1}$ ) was noticed under treatment $\mathrm{T}_{4}(\mathrm{VC} 5 \mathrm{t}$ $\mathrm{ha}^{-1}$ ). During the second year, significantly the maximum available ' $\mathrm{N}$ ' (259 $\left.\mathrm{kg} \mathrm{ha}^{-1}\right)$ in the treatment $\mathrm{T}_{1}$ (FYM $\left.20 \mathrm{t} \mathrm{ha}^{-1}+100 \% \mathrm{RDF}\right)$ and was at par with $\mathrm{T}_{5}(75 \% \mathrm{RDF}+\mathrm{FYM} 20$ $\left.\mathrm{t} \mathrm{ha}^{-1}\right)$. However, the lowest values of $169 \mathrm{~kg}$ $\mathrm{ha}^{-1}$ for available ' $\mathrm{N}$ ' was recorded under the treatment $\mathrm{T}_{4}\left(\mathrm{VC} 5 \mathrm{t} \mathrm{ha}^{-1}\right)$.

There was a significant effect the INM treatments on available ${ }^{2} \mathrm{P}_{2} \mathrm{O}_{5}$ ' in both the years. Mean data of year 2011-12, clearly indicated that the available ' $\mathrm{P}_{2} \mathrm{O}_{5}$ ' varied from 32.3 to $63.5 \mathrm{~kg} \mathrm{ha}^{-1}$. Treatment $\mathrm{T}_{1}$ registered significantly the maximum available ${ }^{2} \mathrm{P}_{2} \mathrm{O}_{5}$ ' $\left(63.5 \mathrm{~kg} \mathrm{ha}^{-1}\right)$. In the year 2012-13, the combined application of FYM $20 \mathrm{t} \mathrm{ha}^{-1}+$ $100 \%$ RDF $\left(\mathrm{T}_{1}\right)$ showed highest available 
' $\mathrm{P}_{2} \mathrm{O}_{5}$ ' (61.9 $\left.\mathrm{kg} \mathrm{ha}^{-1}\right)$. Treatment $\mathrm{T}_{12}$ recorded the lowest available $\mathrm{P}_{2} \mathrm{O}_{5}$ ' $\left(31.0 \mathrm{~kg} \mathrm{ha}^{-1}\right)$.

The data presented revealed a significant effect of INM treatments on ' $\mathrm{K}_{2} \mathrm{O}$ ' during both the years. The values pertaining to available ' $\mathrm{K}_{2} \mathrm{O}^{\prime}$ varied from 273 to $327 \mathrm{~kg} \mathrm{ha}^{-1}$ during 2011-12. The maximum value (327 kg ha-1) was recorded under the treatment of FYM 20 $\mathrm{t} \mathrm{ha}^{-1}+100 \% \mathrm{RDF}\left(\mathrm{T}_{1}\right)$ and was at par with $\mathrm{T}_{5}$ and $\mathrm{T}_{8}$. Second year results (2012-13), the values were found between 270 to $322 \mathrm{~kg}$ ha 1. Significantly higher available ' $\mathrm{K}_{2} \mathrm{O}$ ' (322 $\mathrm{kg} \mathrm{ha}^{-1}$ ) was noticed under the treatment of $\mathrm{T}_{1}$ having combination of FYM $20 \mathrm{t} \mathrm{ha}^{-1}+100 \%$ and was at par with RDF $75 \% \mathrm{RDF}+\mathrm{FYM}$ $20 \mathrm{t} \mathrm{ha}^{-1}\left(\mathrm{~T}_{5}\right)$ and RDF $50 \% \mathrm{RDF}+\mathrm{FYM} 20 \mathrm{t}$ $\mathrm{ha}^{-1}\left(\mathrm{~T}_{8}\right)$.

The higher available $\mathrm{N}, \mathrm{P}_{2} \mathrm{O}_{5}$ and $\mathrm{K}_{2} \mathrm{O}$ in soil after harvest may be due to residual effect of applied nutrients, favourable effect of integrated nutrient management in extracting the various nutrients from soil by crop, greater mineralization of FYM due to synergistic effect of dual inoculation of nitrogen fixer and phosphobacteria (Subbiah, 1992).

\section{Nutrient Content}

The results of both the years as on $\mathrm{N}, \mathrm{P}$ and $\mathrm{K}$ content in tomato fruit as well as in plant are given in Table 3 and 4 . The content of nutrients viz., $\mathrm{N}, \mathrm{P}$ and $\mathrm{K}$ in tomato fruit as well as plant were not significantly affected by any of the INM treatments during both the year.

\section{Nutrient uptake}

Response of different INM treatments on total uptake of nutrients by tomato is furnished in Table 5.The total uptake of nutrients viz., N, P and $\mathrm{K}$ by tomato plant was significantly influenced by the different INM treatments. The significantly higher values of total uptake of $\mathrm{N}\left(136 \mathrm{~kg} \mathrm{ha}^{-1}\right.$ and $\left.145 \mathrm{~kg} \mathrm{ha}^{-1}\right), \mathrm{P}(45.2 \mathrm{~kg}$ $\mathrm{ha}^{-1}$ and $\left.49.8 \mathrm{~kg} \mathrm{ha}^{-1}\right)$ and $\mathrm{K}\left(66 \mathrm{~kg} \mathrm{ha}^{-1}\right.$ and 76 $\mathrm{kg} \mathrm{ha}^{-1}$ ) by tomato were observed with the application of $100 \% \mathrm{RDF}+\mathrm{FYM} 20 \mathrm{t} \mathrm{ha}^{-1}$ $\left(\mathrm{T}_{1}\right)$. In general, the lower values, pertaining to total uptake of these nutrients, were recorded with the treatments receiving only organic manures $\left(\mathrm{T}_{2}, \mathrm{~T}_{3}\right.$ and $\left.\mathrm{T}_{4}\right)$.

The results suggest that the total uptake pattern of nutrients was governed by fruit yield and dry matter production of fruit as well as plant rather than the content of these elements in respective plant parts. Secondly, it could be owing to adequate availability of nutrients for better growth and thereby ultimately resulting in an increased uptake values (Jose et al., 1988). In general, therefore, the efficacy of the inorganic fertilizers was found to be pronounced when they are combined with organic manures (Schuphan, 1974). The higher total content and uptake of plant macro (N, P and $\mathrm{K}$ ) and micro-nutrients ( $\mathrm{Zn}$ and $\mathrm{Fe}$ ) by tomato crop might be obtained due to higher accumulation of all the above nutrients in soil by the application of large amount of chemical fertilizers as well as organic manures. The results are in agreement with those of Patel (2012), Patil (2013) and Mourao et al., (2014).

\section{Yield}

The effect due to various INM treatments on fruit yield per plant and fruit yield per plot are presented in Table 6.Both this character showed significant differences due to different INM treatment in both the years. The first year (2011-12) data indicated that the fruit yield per plant varied from 0.93 to 1.49. Significantly maximum fruit yield per plant $(1.49 \mathrm{~kg})$ was recorded with the combined application of FYM $20 \mathrm{tha}^{-1}+100$ $\%$ RDF $\left(\mathrm{T}_{1}\right)$ and was statistically at par with the treatments $\mathrm{T}_{5}, \mathrm{~T}_{6}$ and $\mathrm{T}_{12}$. 
Table.1 Physico- chemical properties of experimental site

\begin{tabular}{|c|c|c|c|}
\hline Particulars & Initial value & Method employed & Reference \\
\hline Coarse sand $(\%)$ & 1.75 & International pipette method (USDA) & \\
\hline Fine sand $(\%)$ & 9.19 & & Piper,1966 \\
\hline Silt $(\%)$ & 22.94 & & \\
\hline Clay $(\%)$ & 63.71 & & \\
\hline Soil $\mathrm{pH}(1: 2.5$ soil: water ratio) & 7.6 & Potentiometric & Jackson (1973) \\
\hline 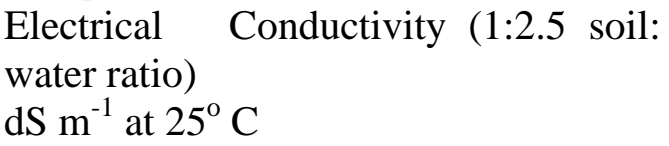 & 0.45 & Conductometric & Jackson (1973) \\
\hline Organic carbon $(\%)$ & 0.60 & Wet-Oxidation & Jackson (1973) \\
\hline $\begin{array}{l}\text { Available 'N' } \\
\left(\mathrm{kg} \mathrm{ha}^{-1}\right)\end{array}$ & 231 & Alkaline Permanganate Oxidation & $\begin{array}{l}\text { Subbiah and Asija } \\
\text { (1956) }\end{array}$ \\
\hline $\begin{array}{l}\text { Available ' } \mathrm{P}_{2} \mathrm{O}_{5} \text { ' } \\
\left(\mathrm{kg} \mathrm{ha}^{-1}\right)\end{array}$ & 33.4 & $\begin{array}{l}\text { Spectro photometric (Extraction with } 0.5 \mathrm{M} \\
\mathrm{NaHCO}_{3}, \mathrm{pH} \text { of 8.5) }\end{array}$ & Olsen et al. (1954) \\
\hline $\begin{array}{l}\text { Available ' } \mathrm{K}_{2} \mathrm{O}^{\prime} \\
\left(\mathrm{kg} \mathrm{ha}^{-1}\right)\end{array}$ & 276 & $\begin{array}{l}\text { Flame photometric (Extraction with } \mathrm{N} \mathrm{NH}_{4} \mathrm{OAc} \\
\text { of } \mathrm{pH} 7.0 \text { ) }\end{array}$ & Jackson (1973) \\
\hline
\end{tabular}


Table.2 Effect of Integrated NutrientManagement onnutrient availability after harvest in soil of tomatocv. "Gujarat Tomato-2"

\begin{tabular}{|c|c|c|c|c|c|c|c|c|c|}
\hline \multirow[t]{3}{*}{ Treatments } & \multicolumn{9}{|c|}{ Available nutrients $\left(\mathrm{kg} \mathrm{ha}^{-1}\right)$} \\
\hline & \multicolumn{3}{|c|}{$\mathbf{N}$} & \multicolumn{3}{|c|}{$\mathbf{P}_{2} \mathbf{O}_{5}$} & \multicolumn{3}{|c|}{$\mathbf{K}_{2} \mathbf{O}$} \\
\hline & 2011-12 & 2012-13 & Pooled & 2011-12 & 2012-13 & Pooled & 2011-12 & $2012-13$ & Pooled \\
\hline $\begin{array}{l}\mathbf{T}_{1}: \text { FYM } 20 \mathrm{tha}^{-1}+100 \% \text { RDF } \\
\quad\left(75: 37.5: 62.5 \text { NPK kg ha }{ }^{-1}\right)\end{array}$ & 255 & 259 & 257 & 63.5 & 61.9 & 62.7 & 327 & 322 & 325 \\
\hline $\mathbf{T}_{\mathbf{2}}$ :Farm Yard Manure $20 \mathrm{t} \mathrm{ha}^{-1}$ & 223 & 252 & 237 & 39.1 & 36.7 & 37.9 & 285 & 279 & 282 \\
\hline $\mathbf{T}_{3}:$ Neem cake $5 \mathrm{t} \mathrm{ha}^{-1}$ & 193 & 211 & 202 & 32.3 & 31.3 & 31.8 & 273 & 270 & 271 \\
\hline $\mathbf{T}_{4}:$ Vermicompost $5 \mathrm{t} \mathrm{ha}^{-1}$ & 161 & 169 & 165 & 37.0 & 36.3 & 36.7 & 274 & 272 & 273 \\
\hline $\mathbf{T}_{\mathbf{5}}: 75 \% \mathrm{RDF}+\mathrm{FYM} 20 \mathrm{t} \mathrm{ha}^{-1}$ & 246 & 248 & 247 & 60.0 & 57.1 & 58.6 & 315 & 312 & 314 \\
\hline $\mathbf{T}_{6}: 75 \% \mathrm{RDF}+\mathrm{NC} 5 \mathrm{t} \mathrm{ha}^{-1}$ & 208 & 239 & 224 & 44.7 & 47.5 & 46.1 & 288 & 281 & 285 \\
\hline $\mathbf{T}_{7}: 75 \% \mathrm{RDF}+\mathrm{VC} 5 \mathrm{t} \mathrm{ha}^{-1}$ & 177 & 184 & 181 & 47.9 & 45.7 & 46.8 & 278 & 275 & 277 \\
\hline $\mathbf{T}_{\mathbf{8}}: 50 \% \mathrm{RDF}+\mathrm{FYM} 20 \mathrm{t} \mathrm{ha}^{-1}$ & 242 & 177 & 209 & 46.0 & 48.3 & 47.2 & 305 & 300 & 302 \\
\hline $\mathbf{T}_{\mathbf{9}}: 50 \% \mathrm{RDF}+\mathrm{NC} 5 \mathrm{t} \mathrm{ha}^{-1}$ & 210 & 234 & 222 & 40.3 & 44.4 & 42.4 & 276 & 273 & 275 \\
\hline $\mathbf{T}_{\mathbf{1 0}}: 50 \% \mathrm{RDF}+\mathrm{VC} 5 \mathrm{tha}^{-1}$ & 173 & 175 & 174 & 43.6 & 43.0 & 43.3 & 276 & 272 & 274 \\
\hline $\mathbf{T}_{11}: 100 \% \mathrm{RDF}+\mathrm{ZnSO}_{4} @ 25 \mathrm{~kg} \mathrm{ha}^{-1}$ & 168 & 174 & 171 & 34.0 & 31.7 & 32.8 & 287 & 282 & 285 \\
\hline $\mathbf{T}_{\mathbf{1 2}}: 100 \% \mathrm{RDF}+\mathrm{FeS}_{4} @ 50 \mathrm{~kg} \mathrm{ha}^{-1}$ & 167 & 172 & 169 & 33.0 & 31.0 & 32.0 & 289 & 285 & 287 \\
\hline S.Em. $( \pm)$ & 6.6 & 6.2 & 4.5 & 2.09 & 2.47 & 1.62 & 9.07 & 9.93 & 6.72 \\
\hline C.D. @ $5 \%$ & 19.3 & 18.2 & 12.9 & 6.1 & 7.2 & 4.6 & 27 & 29 & 19 \\
\hline
\end{tabular}


Table.3 Effect of Integrated Nutrient Management on nutrient content in tomato fruit cv. "Gujarat Tomato-2"

\begin{tabular}{|c|c|c|c|c|c|c|c|c|c|}
\hline \multirow[t]{3}{*}{ Treatments } & \multicolumn{9}{|c|}{ Nutrient content $(\%)$} \\
\hline & \multicolumn{3}{|c|}{$\mathbf{N}$} & \multicolumn{3}{|c|}{$\mathbf{P}$} & \multicolumn{3}{|c|}{$\mathbf{K}$} \\
\hline & 2011-12 & 2012-13 & Pooled & 2011-12 & 2012-13 & Pooled & 2011-12 & 2012-13 & Pooled \\
\hline $\begin{array}{l}\text { T }_{1}: \text { FYM } 20 \mathrm{t} \mathrm{ha}^{-1}+100 \% \text { RDF } \\
\quad\left(75: 37.5: 62.5 \text { NPK kg ha' }{ }^{-1}\right)\end{array}$ & 1.54 & 1.56 & 1.55 & 0.49 & 0.50 & 0.50 & 1.30 & 1.33 & 1.32 \\
\hline $\mathbf{T}_{\mathbf{2}}:$ Farm Yard Manure $20 \mathrm{t} \mathrm{ha}^{-1}$ & 1.45 & 1.46 & 1.46 & 0.44 & 0.48 & 0.46 & 1.25 & 1.28 & 1.27 \\
\hline $\mathbf{T}_{\mathbf{3}}:$ Neem cake $5 \mathrm{t} \mathrm{ha}^{-1}$ & 1.44 & 1.47 & 1.46 & 0.44 & 0.44 & 0.44 & 1.25 & 1.26 & 1.26 \\
\hline $\mathbf{T}_{\mathbf{4}}:$ Vermicompost $5 \mathrm{t} \mathrm{ha}^{-1}$ & 1.44 & 1.47 & 1.46 & 0.45 & 0.43 & 0.44 & 1.25 & 1.27 & 1.26 \\
\hline $\mathbf{T}_{5}: 75 \% \mathrm{RDF}+\mathrm{FYM} 20 \mathrm{tha}^{-1}$ & 1.52 & 1.56 & 1.54 & 0.49 & 0.49 & 0.49 & 1.30 & 1.32 & 1.31 \\
\hline $\mathbf{T}_{6}: 75 \% \mathrm{RDF}+\mathrm{NC} 5 \mathrm{tha}^{-1}$ & 1.51 & 1.55 & 1.53 & 0.48 & 0.49 & 0.49 & 1.30 & 1.32 & 1.31 \\
\hline $\mathbf{T}_{7}: 75 \% \mathrm{RDF}+\mathrm{VC} 5 \mathrm{t} \mathrm{ha}^{-1}$ & 1.51 & 1.55 & 1.53 & 0.48 & 0.48 & 0.48 & 1.29 & 1.31 & 1.30 \\
\hline $\mathbf{T}_{\mathbf{8}}: 50 \% \mathrm{RDF}+\mathrm{FYM} 20 \mathrm{tha}^{-1}$ & 1.49 & 1.53 & 1.51 & 0.47 & 0.48 & 0.47 & 1.29 & 1.30 & 1.29 \\
\hline $\mathbf{T}_{\mathbf{9}}: 50 \% \mathrm{RDF}+\mathrm{NC} 5 \mathrm{t} \mathrm{ha}{ }^{-1}$ & 1.49 & 1.51 & 1.50 & 0.46 & 0.47 & 0.47 & 1.27 & 1.30 & 1.29 \\
\hline $\mathbf{T}_{10}: 50 \% \mathrm{RDF}+\mathrm{VC} 5 \mathrm{tha}^{-1}$ & 1.48 & 1.50 & 1.49 & 0.46 & 0.47 & 0.46 & 1.29 & 1.30 & 1.29 \\
\hline $\mathbf{T}_{11}: 100 \% \mathrm{RDF}+\mathrm{ZnSO}_{4} @ 25 \mathrm{~kg} \mathrm{ha}^{-1}$ & 1.50 & 1.51 & 1.51 & 0.48 & 0.48 & 0.48 & 1.28 & 1.29 & 1.29 \\
\hline $\mathbf{T}_{\mathbf{1 2}}: 100 \% \mathrm{RDF}+\mathrm{FeSO}_{4} @ 50 \mathrm{~kg} \mathrm{ha}^{-1}$ & 1.51 & 1.55 & 1.53 & 0.48 & 0.48 & 0.48 & 1.29 & 1.31 & 1.30 \\
\hline S.Em. $( \pm)$ & 0.03 & 0.04 & 0.03 & 0.02 & 0.02 & 0.01 & 0.025 & 0.029 & 0.019 \\
\hline \multirow[t]{2}{*}{ C.D. @ $5 \%$} & NS & NS & NS & NS & NS & NS & NS & NS & NS \\
\hline & \multicolumn{9}{|c|}{ NS- Non significant } \\
\hline
\end{tabular}


Table.4 Effect of Integrated Nutrient Management on nutrient content in tomato plant cv. "Gujarat Tomato-2"

\begin{tabular}{|c|c|c|c|c|c|c|c|c|c|}
\hline \multirow[t]{3}{*}{ Treatments } & \multicolumn{9}{|c|}{ Nutrient content (\%) in tomato plant cv. "GT-2" } \\
\hline & \multicolumn{3}{|c|}{$\mathbf{N}$} & \multicolumn{3}{|c|}{$\mathbf{P}$} & \multicolumn{3}{|c|}{$\mathbf{K}$} \\
\hline & 2011-12 & 2012-13 & Pooled & 2011-12 & 2012-13 & Pooled & 2011-12 & 2012-13 & Pooled \\
\hline $\begin{array}{l}\mathbf{T}_{1}: \text { FYM } 20 \mathrm{t} \mathrm{ha}^{-1}+100 \% \text { RDF } \\
\quad(75: 37.5: 62.5 \text { NPK kg ha } \\
\end{array}$ & 1.29 & 1.30 & 1.30 & 0.45 & 0.46 & 0.45 & 1.11 & 1.13 & 1.12 \\
\hline $\mathbf{T}_{\mathbf{2}}:$ Farm Yard Manure $20 \mathrm{t} \mathrm{ha}^{-1}$ & 1.19 & 1.22 & 1.20 & 0.40 & 0.41 & 0.41 & 1.06 & 1.07 & 1.06 \\
\hline $\mathbf{T}_{\mathbf{3}}:$ Neem cake $5 \mathrm{t} \mathrm{ha}^{-1}$ & 1.19 & 1.22 & 1.20 & 0.39 & 0.42 & 0.40 & 1.05 & 1.06 & 1.05 \\
\hline $\mathbf{T}_{\mathbf{4}}:$ Vermicompost5 $\mathrm{t} \mathrm{ha}^{-1}$ & 1.18 & 1.20 & 1.19 & 0.39 & 0.40 & 0.39 & 1.06 & 1.07 & 1.07 \\
\hline $\mathbf{T}_{\mathbf{5}}: 75 \% \mathrm{RDF}+\mathrm{FYM} 20 \mathrm{t} \mathrm{ha}^{-1}$ & 1.27 & 1.30 & 1.28 & 0.44 & 0.45 & 0.44 & 1.10 & 1.11 & 1.11 \\
\hline $\mathbf{T}_{\mathbf{6}}: 75 \% \mathrm{RDF}+\mathrm{NC} 5 \mathrm{tha}^{-1}$ & 1.27 & 1.30 & 1.28 & 0.43 & 0.45 & 0.44 & 1.09 & 1.10 & 1.10 \\
\hline $\mathbf{T}_{7}: 75 \% \mathrm{RDF}+\mathrm{VC} 5 \mathrm{t} \mathrm{ha}{ }^{-1}$ & 1.25 & 1.28 & 1.26 & 0.42 & 0.44 & 0.43 & 1.08 & 1.10 & 1.09 \\
\hline $\mathbf{T}_{\mathbf{8}}: 50 \% \mathrm{RDF}+\mathrm{FYM} 20 \mathrm{t} \mathrm{ha}^{-1}$ & 1.24 & 1.24 & 1.24 & 0.42 & 0.44 & 0.43 & 1.08 & 1.09 & 1.08 \\
\hline $\mathbf{T}_{\mathbf{9}}: 50 \% \mathrm{RDF}+\mathrm{NC} 5 \mathrm{t} \mathrm{ha}^{-1}$ & 1.23 & 1.24 & 1.23 & 0.42 & 0.43 & 0.42 & 1.08 & 1.09 & 1.08 \\
\hline $\mathbf{T}_{\mathbf{1 0}}: 50 \% \mathrm{RDF}+\mathrm{VC} 5 \mathrm{tha}^{-1}$ & 1.21 & 1.23 & 1.22 & 0.41 & 0.42 & 0.42 & 1.07 & 1.08 & 1.08 \\
\hline $\mathbf{T}_{11}: 100 \% \mathrm{RDF}+\mathrm{ZnSO}_{4} @ 25 \mathrm{~kg} \mathrm{ha}^{-1}$ & 1.26 & 1.27 & 1.26 & 0.42 & 0.44 & 0.43 & 1.08 & 1.09 & 1.08 \\
\hline $\mathbf{T}_{\mathbf{1 2}}: 100 \% \mathrm{RDF}+\mathrm{FeSO}_{4} @ 50 \mathrm{~kg} \mathrm{ha}^{-1}$ & 1.26 & 1.28 & 1.27 & 0.43 & 0.44 & 0.44 & 1.08 & 1.08 & 1.08 \\
\hline S.Em. $( \pm)$ & 0.04 & 0.04 & 0.03 & 0.02 & 0.02 & 0.01 & 0.033 & 0.028 & 0.021 \\
\hline C.D. @ $5 \%$ & NS & NS & NS & NS & NS & NS & NS & NS & NS \\
\hline
\end{tabular}


Table.5 Effect of Integrated Nutrient Management on total nutrient uptake of tomato cv. "Gujarat Tomato-2"

\begin{tabular}{|c|c|c|c|c|c|c|c|c|c|}
\hline \multirow[t]{3}{*}{ Treatments } & \multicolumn{9}{|c|}{ Nutrient uptake $\left(\mathrm{kg} \mathrm{ha}^{-1}\right)$} \\
\hline & \multicolumn{3}{|c|}{$\mathbf{N}$} & \multicolumn{3}{|c|}{$\mathbf{P}$} & \multicolumn{3}{|c|}{$\mathbf{K}$} \\
\hline & 2011-12 & 2012-13 & Pooled & 2011-12 & 2012-13 & Pooled & 2011-12 & 2012-13 & Pooled \\
\hline $\begin{array}{l}\text { T }_{1}: \text { FYM } 20 \mathrm{tha}^{-1}+100 \% \text { RDF } \\
\quad\left(75: 37.5: 62.5 \mathrm{NPK} \mathrm{kg} \mathrm{ha}{ }^{-1}\right)\end{array}$ & 136 & 145 & 140 & 45.2 & 49.8 & 47.5 & 66 & 76 & 71 \\
\hline $\mathbf{T}_{\mathbf{2}}:$ Farm Yard Manure $20 \mathrm{t} \mathrm{ha}^{-1}$ & 97 & 104 & 100 & 31.9 & 34.7 & 33.3 & 45 & 49 & 47 \\
\hline $\mathbf{T}_{\mathbf{3}}:$ Neem cake $5 \mathrm{t} \mathrm{ha}^{-1}$ & 94 & 98 & 96 & 30.0 & 32.4 & 31.2 & 43 & 47 & 45 \\
\hline $\mathbf{T}_{\mathbf{4}}:$ Vermicompost $5 \mathrm{t} \mathrm{ha}^{-1}$ & 88 & 92 & 90 & 28.4 & 29.6 & 29.0 & 41 & 44 & 42 \\
\hline $\mathbf{T}_{5}: 75 \% \mathrm{RDF}+\mathrm{FYM} 20 \mathrm{tha}^{-1}$ & 130 & 140 & 135 & 43.9 & 47.3 & 45.6 & 64 & 71 & 67 \\
\hline $\mathbf{T}_{6}: 75 \% \mathrm{RDF}+\mathrm{NC} 5 \mathrm{t} \mathrm{ha}^{-1}$ & 127 & 135 & 131 & 42.7 & 45.3 & 44.0 & 62 & 66 & 64 \\
\hline $\mathbf{T}_{7}: 75 \% \mathrm{RDF}+\mathrm{VC} 5 \mathrm{t} \mathrm{ha}^{-1}$ & 119 & 125 & 122 & 39.4 & 41.6 & 40.5 & 56 & 60 & 58 \\
\hline $\mathbf{T}_{\mathbf{8}}: 50 \% \mathrm{RDF}+\mathrm{FYM} 20 \mathrm{t} \mathrm{ha}^{-1}$ & 113 & 116 & 114 & 37.5 & 39.7 & 38.6 & 53 & 57 & 55 \\
\hline $\mathbf{T}_{\mathbf{9}}: 50 \% \mathrm{RDF}+\mathrm{NC} 5 \mathrm{tha}^{-1}$ & 108 & 114 & 111 & 36.2 & 38.4 & 37.3 & 50 & 55 & 53 \\
\hline $\mathbf{T}_{10}: 50 \% \mathrm{RDF}+\mathrm{VC} 5 \mathrm{tha}^{-1}$ & 103 & 109 & 106 & 34.4 & 36.4 & 35.4 & 49 & 53 & 51 \\
\hline $\mathbf{T}_{11}: 100 \% \mathrm{RDF}+\mathrm{ZnSO}_{4} @ 25 \mathrm{~kg} \mathrm{ha}^{-1}$ & 121 & 125 & 123 & 39.9 & 42.3 & 41.1 & 58 & 62 & 60 \\
\hline $\mathbf{T}_{\mathbf{1 2}}: 100 \% \mathrm{RDF}+\mathrm{FeSO}_{4} @ 50 \mathrm{~kg} \mathrm{ha}^{-1}$ & 125 & 129 & 127 & 41.7 & 43.1 & 42.4 & 60 & 63 & 61 \\
\hline S.Em. $( \pm)$ & 6.6 & 4.5 & 4.0 & 1.5 & 2.3 & 1.4 & 2.1 & 3.0 & 1.8 \\
\hline C.D. @ $5 \%$ & 19.3 & 13.3 & 11.4 & 4.5 & 6.7 & 3.9 & 6.2 & 8.7 & 5.2 \\
\hline
\end{tabular}


Table.6 Effect of Integrated Nutrient Management on yield of tomato cv. "Gujarat Tomato-2"

\begin{tabular}{|c|c|c|c|c|c|c|}
\hline \multirow[t]{2}{*}{ Treatments } & \multicolumn{3}{|c|}{ Fruit yield per plant (kg) } & \multicolumn{3}{|c|}{ Fruit yield per plot (kg) } \\
\hline & 2011-12 & 2012-13 & Pooled & 2011-12 & 2012-13 & Pooled \\
\hline $\begin{array}{l}\mathbf{T}_{1}: \text { FYM } 20 \mathrm{tha}^{-1}+100 \% \text { RDF } \\
\quad\left(75: 37.5: 62.5 \mathrm{NPK} \mathrm{kg} \mathrm{ha}{ }^{-1}\right)\end{array}$ & 1.49 & 1.58 & 1.54 & 29.86 & 31.56 & 30.71 \\
\hline $\mathbf{T}_{\mathbf{2}}:$ Farm Yard Manure $20 \mathrm{t} \mathrm{ha}^{-1}$ & 0.98 & 1.04 & 1.01 & 19.65 & 20.82 & 20.24 \\
\hline $\mathbf{T}_{\mathbf{3}}:$ Neem cake $5 \mathrm{t} \mathrm{ha}^{-1}$ & 0.97 & 1.01 & 0.99 & 19.33 & 20.26 & 19.79 \\
\hline $\mathbf{T}_{\mathbf{4}}:$ Vermicompost $5 \mathrm{t} \mathrm{ha}^{-1}$ & 0.93 & 0.99 & 0.96 & 18.67 & 19.72 & 19.19 \\
\hline $\mathbf{T}_{5}: 75 \% \mathrm{RDF}+\mathrm{FYM} 20 \mathrm{t} \mathrm{ha}^{-1}$ & 1.47 & 1.51 & 1.50 & 29.31 & 30.10 & 29.71 \\
\hline $\mathbf{T}_{\mathbf{6}}: 75 \% \mathrm{RDF}+\mathrm{NC} 5 \mathrm{t} \mathrm{ha}^{-1}$ & 1.41 & 1.44 & 1.43 & 28.18 & 28.88 & 28.53 \\
\hline $\mathbf{T}_{7}: 75 \% \mathrm{RDF}+\mathrm{VC} 5 \mathrm{t} \mathrm{ha}^{-1}$ & 1.21 & 1.28 & 1.25 & 24.21 & 25.69 & 24.95 \\
\hline $\mathbf{T}_{\mathbf{8}}: 50 \% \mathrm{RDF}+\mathrm{FYM} 20 \mathrm{t} \mathrm{ha}^{-1}$ & 1.12 & 1.23 & 1.17 & 22.30 & 24.59 & 23.44 \\
\hline $\mathbf{T}_{\mathbf{9}}: 50 \% \mathrm{RDF}+\mathrm{NC} 5 \mathrm{t} \mathrm{ha}^{-1}$ & 1.07 & 1.17 & 1.12 & 21.38 & 23.35 & 22.37 \\
\hline $\mathbf{T}_{\mathbf{1 0}}: 50 \% \mathrm{RDF}+\mathrm{VC} 5 \mathrm{t} \mathrm{ha}^{-1}$ & 1.03 & 1.13 & 1.08 & 20.56 & 22.53 & 21.54 \\
\hline $\mathbf{T}_{11}: 100 \% \mathrm{RDF}+\mathrm{ZnSO}_{4} @ 25 \mathrm{~kg} \mathrm{ha}^{-1}$ & 1.26 & 1.36 & 1.31 & 25.43 & 27.18 & 26.31 \\
\hline $\mathbf{T}_{\mathbf{1 2}}: 100 \% \mathrm{RDF}+\mathrm{FeSO}_{4} @ 50 \mathrm{~kg} \mathrm{ha}^{-1}$ & 1.32 & 1.40 & 1.36 & 26.32 & 27.98 & 27.15 \\
\hline S.Em. $( \pm)$ & 0.07 & 0.07 & 0.04 & 1.47 & 1.34 & 0.90 \\
\hline C.D. @ $5 \%$ & 0.22 & 0.20 & 0.13 & 4.31 & 3.93 & 2.54 \\
\hline
\end{tabular}


The minimum fruit yield per plant $(0.93 \mathrm{~kg})$ was noticed in the application of organic manure treatment i.e. VC $5 \mathrm{t} \mathrm{ha}^{-1}\left(\mathrm{~T}_{4}\right)$. The values for this character during second year (2012-13) varied from 0.99 to 1.58 . The trend of treatments was found similar to that of the preceding year results.

With respect to first year data, the fruit yield per plot varied from 18.67 to $29.86 \mathrm{~kg}$. Significantly maximum fruit yield $(29.86 \mathrm{~kg})$ per plot was achieved in the treatment treated with FYM $20 \mathrm{t} \mathrm{ha}^{-1}+100 \% \operatorname{RDF}\left(\mathrm{T}_{1}\right)$. In second year statistical data, the fruit yield per plot was noticed from 19.72 to $31.56 \mathrm{~kg}$. The data showed that significantly the maximum fruit yield $(31.56 \mathrm{~kg})$ per plot was noticed in the treatment $T_{1}$ receiving the combination of FYM $20 \mathrm{t} \mathrm{ha}^{-1}+100 \%$ RDF; however it was at par with the treatments like $\mathrm{T}_{5}, \mathrm{~T}_{6}$ and $\mathrm{T}_{12}$. The treatment $\mathrm{T}_{4}$ consisting only organic manure in the form of $\mathrm{VC} 5 \mathrm{t} \mathrm{ha}^{-1}$ recorded the lowest fruit yield per plot $(19.72 \mathrm{~kg})$.

The yield attributes of tomato is closely associated with growth components. Higher number of fruits and fruit weight may be due to increased growth components of tomato plant at RDF and organic manure along. This might have helped in producing higher amount of carbohydrates which might have translocated from source (leaf) to reproductive parts (sink) resulting in more number of fruits and fruit weight. The increase in the tomato yield may also be attributed to the higher absorption of $\mathrm{N}, \mathrm{P}$ and $\mathrm{K}$ which might have favourably affected the chlorophyll content of leaves resulting increased synthesis of carbohydrates and build up of new cells (Jagadeesha, 2008). The yield and yield attributing characters were better due to plants which were supplied nutrients from chemical fertilizers and organic manures that were readily available to plants in sufficient amount throughout the growth period (Islam et al., 2013).
In conclusion, in the light of the results obtained from this investigation, it can be concluded that the efficacy of the inorganic fertilizers was pronounced when they are combined with organic manures.

\section{References}

Chadha, K.L. 2002. "Handbook of Horticulture", Indian Council of Agricultural Research, New Delhi, India, pp. 1-64.

Islam, M.R., Chaudhary, M.A.H., Saha, B.K. and Hasan, M.M. 2013. Integrated nutrient management on soil fertility, growth and yield of tomato. $J$. Bangladesh Agril. Univ., 11(1): 33-40.

Jagadeesha, V. 2008. Effect of organic manures and bio-fertilizers on growth, seed yield and quality in tomato (Lycopersicon esculentum Mill.) cv. 'Megha', Thesis M.Sc. University of Agricultural Sciences, Dharwad.

Jose, D., Shanmugavelu, K.G. and Thamburaj, S. 1988. Studies on the efficacy of organic $v s$ inorganic form of nitrogen in brinjal. Ind. J. Hort., 45(1\& 2): 100-104.

Mourao, I., Pinto, R., Brito, L.M. and Countinho, J. 2014. Effect of organic fertilizers on yield and quality of green house organic tomato.

Olsen, S.R., Cole, C.V., Watanabe, F.S. and Dean, L.A. 1954. Estimation of available phosphorus in soils by extraction with sodium bicarbonate. U.S. Dept. of Agric. Circ., 939.

Panse, V.G. and Sukhatme, P.V. 1954. Statistical methods for agricultural workers, Indian Council of Agricultural Research.

Patel, P.S. 2012. Effect of different proportion of organics on productivity of pit planted sugarcane under organic farming system. Thesis Ph.D. Navsari Agricultural University, Navsari, Gujarat. 
Patil, T.D. 2013. Effect of rates of castor cake and banana pseudostem sap on yield and quality of organically grown garlic. Thesis Ph.D. Navsari Agricultural University, Navsari, Gujarat.

Piper, C.S. 1966. Soil and Plant Analysis. Hans Publisher, Bombay.

Schuphan, W. 1974. Nutritional value of crops as influence by organic and inorganic fertilizer treatment. Results of 12 years experiments with vegetables
(1960-1972. Qual. Plant-Pl. Fds. Hum. Nut., 23(4): 333-358.

Subbiah, B.V. and Asija, G.L. 1956. A rapid procedure for estimation of available nitrogen in soils. Curr. Sci., 25: 259260.

Subbiah, K. 1992. Effect of nitrogen and Azospirillium on yield and nutrient uptake in tomato. Madras Agr. J., 9(10): 600-604.

\section{How to cite this article:}

Tekale, G. S., S. N. Saravaiya, P. B. Jadhav, C. D. Tekale and Patel, R. P. 2017. Integrated Nutrient Management (INM) on Nutrient Availability, Uptake and Yield of Tomato (Lycopersicon esculentum Mill.) cv. "Gujrat Tomato-2". Int.J.Curr.Microbiol.App.Sci. 6(5): 864-874. doi: https://doi.org/10.20546/ijcmas.2017.605.097 